\section{Pseudolymphoma tattoo-induced}

\section{Giorgio Pasolini, ${ }^{1}$ Patrizia Ghidini', Mariachiara Arisi, ${ }^{1}$ Alessandra Pedretti, ${ }^{1}$ Marco Ungari² \\ Piergiacomo Calzavara Pinton ${ }^{1}$ \\ 'Department of Dermatology and 2Department of Pathology, Spedali Civili di Brescia, University of Brescia, Italy}

\section{Abstract}

Tattooing has become more and more popular in today's society. The most common dermatological tattoo complications are represented by hypersensitivity reaction to tattoo pigments like irritant and allergical contact dermatitis, development of lichenoid areas and granulomatous responses, such as sarcoid granulomas or foreign body granulomas. Less frequently patients developing discoid lupus erythematous have been reported. Pseudolymphoma is an uncommon reactive lymphocytic proliferation mimicking the histological and clinical features of a malignant skin lymphoma. We herein report a pseuldoymphoma limited to the red area of a multicolour tattoo of the leg.

\section{Case Report}

A 34-year-old man presented with a six months history of mildly itching plaque of the left leg that he developed two months after the injection of a tattoo (Figure 1). The patient was otherwise in good health and he had no personal or family history of allergic diseases or contact dermatitis. At dermatological examination, we observed a 12 square centimeters persistent swelling of the red area of a multicolour (red, black, green, yellow and blue) tattoo of the left leg. There was no involvement of the regional lymphonodes. A $4 \mathrm{~mm}$ punch skin biopsy was performed and histopathological examination showed acanthosis, enlarged interpapillary ridges and compact ortho-hyperkeratosis overlying a dense dermal infiltrate of lymphocytes of small and moderate size without nuclear atypia, sometimes grouped in clusters and with exocitosis. Scattered macrophages with small intracytoplasmic granules of brown pigment, fibrous reaction and focal erythrocyte extravasations were seen as well (Figure 2). On immunohistochemical analysis the lymphoid infiltrate showed a CD3 + CD4 + phenotype, with scattered CD20 + B lymphoid cells (Figure 3 ). CD30 + large cells were not detected. The plasma cell population showed a polytypical pattern of immunoglobulin lightchains. The histological architectural pattern suggested a diagnosis of T-cell pseudolymphoma.

Patch tests with the Standard series recommended by the Italian Society of Occupational and Environmental Allergological Dermatology (SIDAPA) and with substances often present into tattoo dyes (ammonium chloride mercury, sulphate mercury (cinnabar), cadmium, copper, titanium, iron, chromium sulphate, chromium chloride, 2-[ethyl[4-[(4-nitrophenyl)azo] phenyl] amino],4-(4-Nitrophen ylazo)aniline, Ethyl (2-mercaptobenzoato-S) mercury sodium salt, paraphenylendiamine ) showed a strong erythematous vesicular reaction (3+) to ammonium chloride mercury, cinnabar and Thimerosal after 48 hours.

The patient refused the surgical removal of the tattoo. A topical therapy with clobetasol dipropionate twice daily was prescribed and at a three months follow-up visit the lesion appeared unchanged.

\section{Discussion}

Tattoing has become a common custom all over the world. Complications deriving from body tattoos are relatively uncommon if we think to the whole number of persons that recurs to this technique; they can consist in irritant and allergic contact dermatitis to tattoo dyes, development of lichenoid reactions and granulomatous responses such as sarcoid granulomas or foreign body granulomas. ${ }^{1,2}$ Also cases of discoid lupus erithematous have been reported. ${ }^{3}$

Skin pseudolymphoma is a reactive proliferation of benign lymphocytes mimicking the histological and clinical features of a malignant lymphoma. ${ }^{2}$

The pathogenetic mechanisms are unclear. Pseudolymphomas can be secondary to medications, arhtropod bites, borrelia infections, vaccines, UV light and tattoo dyes and they may be caused by persistent allergic contact dermatitis or to the subcutaneous injection of allergens. ${ }^{4}$ Tattoo-induced pseudolymphomas are rare with fifteen cases reported so far. ${ }^{1,2,5}$ In these cases dye pigments in the dermis act as an antigen stimulus determining a proliferation of lymphoid cells; ${ }^{5}$ they can appear from few months to 6 years after a tattoo placement. ${ }^{5}$ The most cases were described following red tattoos in patients with delayed contact sensitivity to Cinnabar (Mercuric Sulphate) but pseudolymphomas can occur also in blue (mainly cobalt salts) or green (mainly chrome salts) areas of tattoos. ${ }^{6}$ In the present case, a strong reaction to Cinnabar has been found together with positivities to other red pigments (e.g. red one disperse, ammonium chlo-
Correspondence: Mariachiara Arisi, Department of Dermatology, Spedali Civili di Brescia, P.le Spedali Civili 1, 25123 Brescia, Italy.

Tel. +39.030.399.5300 - Fax. +39.030.399.53015.

E-mail: mariachiara.arisi@gmail.com

Key words: pseudolymphoma, tattoo, allergic contact dermatitis.

Received for publication: 18 July 2011

Revision received: 14 October 2011.

Accepted for publication: 24 October 2011.

This work is licensed under a Creative Commons Attribution NonCommercial 3.0 License (CC BYNC 3.0).

(C) Copyright G. Pasolini et al., 2011

Licensee PAGEPress, Italy

Dermatology Reports 2011; 3:e47

doi:10.4081/dr.2011.e47

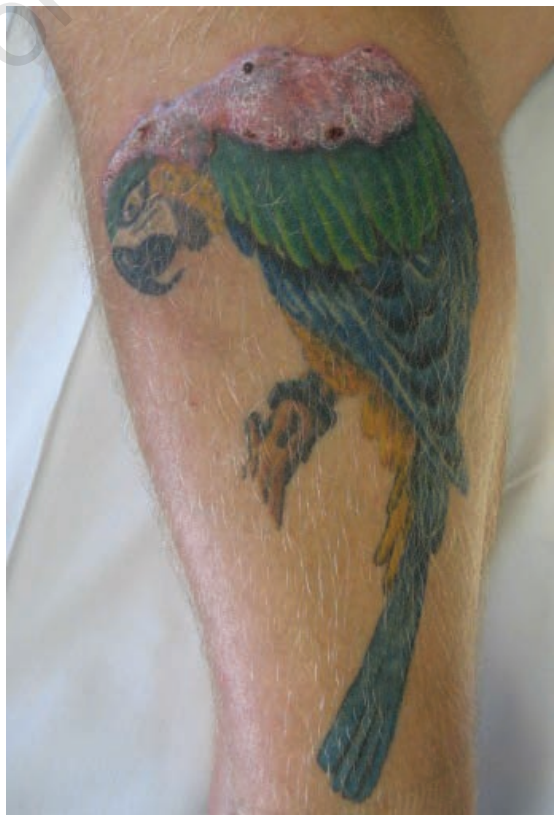

Figure 1. Mildly itching plaque of the left leg.

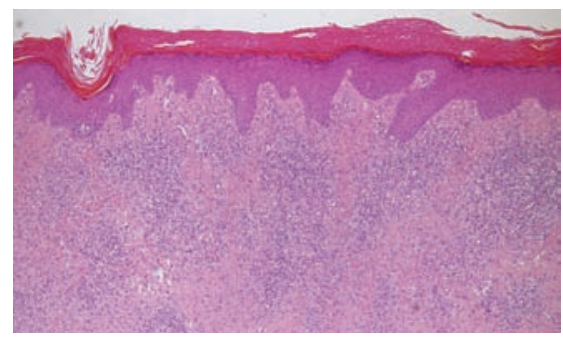

Figure 2. Scattered macrophages with small intracytoplasmic granules of brown pigment, fibrous reaction and focal erythrocyte extravasations. 
ride mercury) and thimerosal as preservative.

Tattoo pseudolymphomas have been traeted with topical or intralesional corticosteroids, surgical excision or laser treatment. ${ }^{7}$ A complete regression of the skin lesions has been described also after the assumption of hydroxycloroquine sulphate ${ }^{6}$ and a spontaneous remission of the disease has also been reported. ${ }^{5}$ In our case, at three months follow up skin lesions remained substantially unchanged

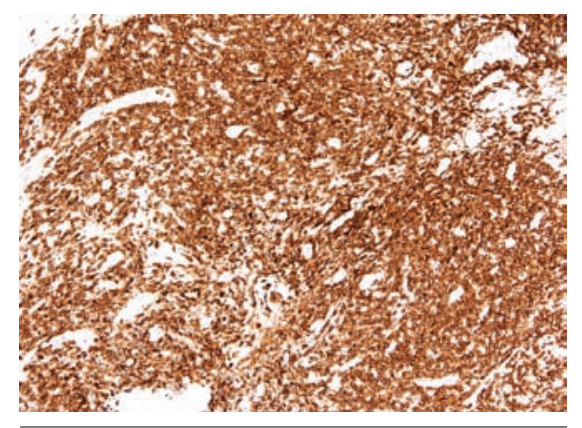

Figure 3. Immunohistochemical analysis showed a $\mathrm{CD3}^{+}$and $\mathrm{CD}^{+}$lymphoid phenotype despite a topical corticosteroid therapy and the patient was lost at follow-up.

Altought pseudolymphoma is considered a benign disease ${ }^{7}$ a prolonged follow up is mandatory because of the reported hazard of progression of cutaneous pseudolymphoma to lymphoma. Sangueza et al. described the progression of a tattoo- induced T-pseudolymphoma, with 10-20\% B-cells and characteristics of benign hystology, into a malignant monoclonal B-cell large lymphoma. ${ }^{8}$

\section{References}

1. Sowden JM, Byrne JP, Smith AG, et al. Red tattoo reactions: X-ray microanalysis and patch-test studies. Br J Dermatol 1991; 124:576-80.

2. Shin JB, Seo SH, Kim BK, Kim IH. Delayed Cutaneous T cell pseudolymphoma at the site of a semipermanent lip-liner tattoo. Dermatology 2009;218:75-8.

3. Kazandjieva J, Tsankov N. Tattoos: derma- tological complications. Clin Dermatol 2007;25:375-82.

4. Kuo WE, Richwine EE, Sheehan DJ. Pseudolymphomatous and lichenoid reaction to a red tattoo: a case report. Cutis 2011;87:89-92.

5. Gutermuth J, Hein R, Fend F, Ring J. Cutaneous Pseudolymphoma arising after tattoo placement. J Eur Acad Dermatol Venereol 2007;21:536-78.

6. Patrizi A, Raone B, Savoia F, et al. Tattooinduced Psudolymphomatous reaction and its Successful Treatment with Hydroxychloroquine. Acta derm Venereol 2009; 89:327-8.

7. Chiang C, Romero L. Cutaneous Lymphoid Hyperplasia (Pseudolymphoma) in a Tattoo After Far infrared Light. Dermatologic Surg 2009;35:1434-8.

8. Sangueza OP, Yadav S, White CR Jr. Evolution of B-cell lymphoma from pseudolymphoma. A multidisciplinary approach using histology, immunohistochemistry, and Southern blot analysis. Am J Dermatopathol 1992;14:408-13. 